Teaching
Methods

\title{
A Software Tool for Teaching Backcross Breeding Simulation
}

\author{
Ana Fita ${ }^{1,3}$, Néstor Tarín ${ }^{2}$, Jaime Prohens ${ }^{1}$, and \\ Adrián Rodríguez-Burruezo ${ }^{1}$
}

AdDitional INDEX WORDs. Cucumis melo, effective learning, breeding methods, Retromelon

\begin{abstract}
SUMMARY. Plant breeding programs involve multiple steps, and their complete development cannot be experienced by students in a single academic year. Tools of communication and information technologies offer a great opportunity to improve students' skills, but only a few software are available for training undergraduate students in plant breeding programs. Here we present a student-friendly software tool, Retromelon, for simulating the backcross breeding method. We also evaluate its suitability and performance as a learning tool. This software is designed to recreate a real melon (Cucumis melo) backcross breeding program aimed at introgressing the resistance to melon vine decline from the tolerant melon cultivar Pat81 into the susceptible cultivar Piel de Sapo. The software simulates the most important steps and decisions that must be made by a plant breeder in a backcross breeding program, including the number of individuals to evaluate in each generation, evaluation of agronomic traits, and selection of individuals. This software is being used by undergraduate horticulture students. Our results have shown that the use of this tool in the classroom increases the motivation of the students, and therefore, benefits the learning process. This software is available upon request both in English and Spanish versions.
\end{abstract}

$\mathrm{P}$ lant breeding is an exciting agricultural discipline as it integrates a broad range of fields of study, such as genetics, crop science, horticulture, botany, biochemistry, molecular biology, pathology, entomology, and statistics. Because of the comprehensive features of plant breeding, students may find it difficult to understand the basic concepts and approaches of plant breeding programs, which are frequently long and complex processes involving

\footnotetext{
${ }^{1}$ Departamento de Biotecnología, Universidad Politécnica de Valencia, Camino de Vera 14, Valencia 46022, Spain

${ }^{2}$ IN2, Cortes Valencianas 39, Valencia 46015, Spain ${ }^{3}$ Corresponding author. E-mail: anfifer@btc.upv.es.
}

multiple steps and decisions. Without an integrative view of the whole discipline, students may devote most of their efforts to the study of individual units and might end up without a cohesive understanding of the whole process of breeding programs. In some cases, students may lose interest in the subject and consequently may fail in their courses. In this respect, one of the most effective ways to integrate ideas and favor students' ability to consolidate their knowledge is based on complementing the theory with activities that come close to real-world practices (St. Hilaire et al., 2009).

Laboratory sessions, along with class activities, are considered to be very important in the efficient and successful teaching of genetics and plant breeding (Rodríguez-Burruezo et al., 2009a). Of the six European Credit Transfer System (ECTS) credits given for one semester of the degrees in Technical Engineer in Horticulture and Gardening and Engineer in Agronomy at the Universidad Politécnica de Valencia, Valencia, Spain (roughly corresponding to $60 \mathrm{~h}$ of teaching sessions), three ECTS credits correspond to lectures and three correspond to laboratory or glasshouse sessions. The laboratory and glasshouse sessions include contact with and utilization of plant materials in genetics and plant breeding protocols: observation of mitosis and meiosis, Mendelian genetics, genetic resources characterization, wild relatives and domestication, improvement and adaptation of new crops, floral biology, pollen viability, hybridization, variation and evaluation of traits of agronomic interest, in vitro culture, and molecular analysis (Prohens et al., 2010). These sessions are especially useful for presenting and applying short protocols or techniques and are relatively easy to prepare and perform, even with a large number of students.

Unfortunately, most plant breeding programs typically take 5 to 10 years to release a new cultivar (Acquaah, 2007; Rodríguez-Burruezo et al., $2009 \mathrm{~b}$ ). This factor makes it virtually impossible to carry out a full breeding program in one semester, and one can only perform the initial processes involved. Even though lecturers can explain how mating and selection are performed in a breeding program, our teaching experience indicates that undergraduate students may experience serious difficulties in having an overall vision of the whole process. Consequently, students usually do not pay enough attention to relevant technical and practical issues that are critical for the success of a plant breeding program. This lack of attention is particularly true for those aspects related to the selection of plants for mating, the importance of the genetic control of the trait(s) of interest, the number of individuals to be evaluated in each generation, the propagation material to be used for the next generation, and the importance of taking care to avoid mistakes in the crosses. Our aim is for students to understand the methodologies and processes involved in plant breeding programs and the real day-to-day work of a plant breeder. 
Without any illustrative practice of these programs, students are often unable to gain this understanding.

E-learning environments have several advantages for teaching complex processes like plant breeding: they reduce the time required to reach a specified level of learning, they allow pauses that facilitate the assimilation of processes, they provide virtual experiences with a lower degree of risk than laboratory or glasshouse classes, and they enable analysis of the decision-making process as opposed to a mere analysis of results (Lea-Cox et al., 2010; Nakhlek and Krajcik, 1993). All these advantages make these systems especially suitable for teaching complex or long-term processes. Although the number of interactive programs available for the teaching of plant breeding is growing fast, with software such as Breeding Game, an intuitive introduction to plant breeding, and Pedimap, a software program for visualizing genetic and phenotypic data in pedigrees of the Wageningen University and Research Center (2010), there is still a lack of student-friendly software tools that simulate plant breeding programs, particularly at the undergraduate level.

Here we describe the software program Retromelon, which, using the specific case of breeding melon, is a tool aimed at teaching the backcross breeding method to undergraduate horticulture students. The students' learning experience using this software is also discussed.

\section{Materials and methods}

Software DeVelopment. The Retromelon software simulates backcross breeding programs, which are amongst the most frequently used breeding methods (Acquaah, 2007; Rodríguez-Burruezo et al., 2009b). Retromelon was written in VisualBasic (Microsoft, Redmond, WA), and it presents a series of interactive interfaces in which the student has to make decisions about the breeding program. The software generates the offspring of each cross according to the theoretical expected values for both the recovery of the genetic background of recurrent parent $(\operatorname{Pr})$ and the genetic configuration of the trait of interest from the donor parent $(\mathrm{Pd})$. For example, when the genetic control of the trait of interest is dominant $(R>r$; resistance $>$ susceptibility) and the parents are homozygous for the trait $(\operatorname{Pr}=r r$ and susceptible; $\mathrm{Pd}=R R$ and resistant), all the individuals of the $F_{1}$ generation will be resistant (genotype $R r$ ) and will have $50 \% \operatorname{Pr}$ genome recovery.

The software includes a series of pictures to illustrate the presence of the trait of interest and the agronomic trait recovery of the recurrent parent. The pictures used were taken from a real backcross breeding program performed by Fita et al. (2009a, b). This breeding program was aimed at introgressing the resistance to melon vine decline (MVD), a root rot fungal disease caused by Monosporacus cannonballus, from the wild melon Cucumis melo ssp. agrestis 'Pat 81 ' into the susceptible C. melo ssp. melo 'Piel de Sapo'. Therefore, the trait of interest in the software is the resistance to MVD; resistant plants show healthy and vigorous root systems in the presence of the pathogen as opposed to susceptible plants, which have severely affected and rotted roots. In addition, to illustrate the recovery of Pr agronomic appearance, we have included pictures of melon fruit from successive backcross generations. The progeny become genetically and phenotypically more similar to the recurrent parent after each backcross generation. Because it is impossible to display all the possible combinations among segregating traits, the illustration of the recovery of the $\mathrm{Pr}$ traits in the software has been limited to the morphological fruit features (apparent fruit quality), with a numerical indication of the theoretical recovery of the genetic background of the recurrent parent according to the backcross generation involved. The program includes the generation of a final report, designed with Crystal Reports (SAP; Walldorf, BadenWürttemberg, Germany), in which the student can see the results of the program.

Procedure of USe. The software is designed to recreate the relevant steps of a backcross breeding program aimed at introgressing a monogenic trait. Given that a breeding program is run differently if the trait is controlled by a dominant as opposed to a recessive allele, the first step when running Retromelon is to select the genetic control of the trait of interest (Fig. 1). MVD tolerance is controlled by a major dominant gene (Fita et al., 2007). Nevertheless, for teaching purposes, we have introduced two possible alternatives (i.e., working with a resistance trait controlled by a dominant or a recessive allele).

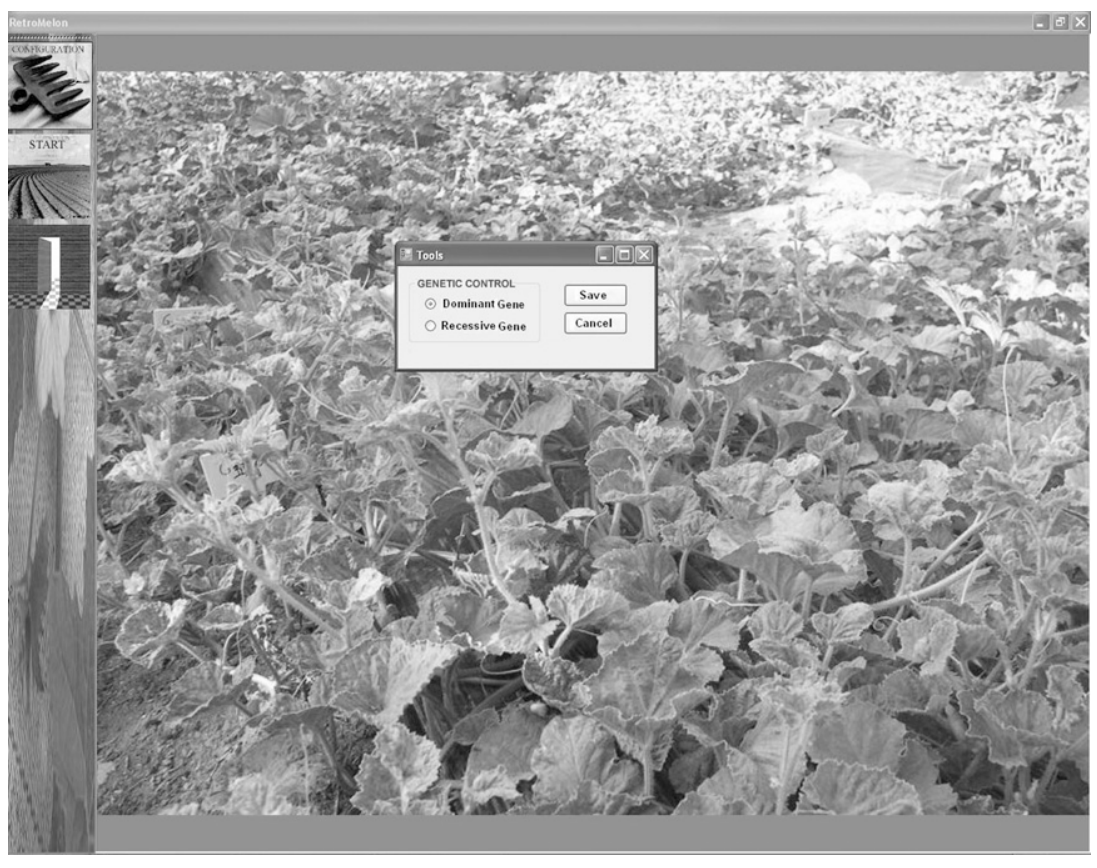

Fig. 1. Retromelon software screenshot showing the starting point of the program. At this stage, the student must set the genetic control of the gene. The software is prepared to work with a trait controlled by a dominant or a recessive allele. 
Once the genetic control is chosen, students must perform the first cross. At this step, the students are requested to indicate which genotype is going to be used as the female parent and which as the male parent, and how many individuals of the first filial generation $\left(\mathrm{F}_{1}\right)$ offspring will be evaluated (Fig. 2). It is worthwhile to note that in each generation it is possible to choose between crossing two plants or self-pollinate.

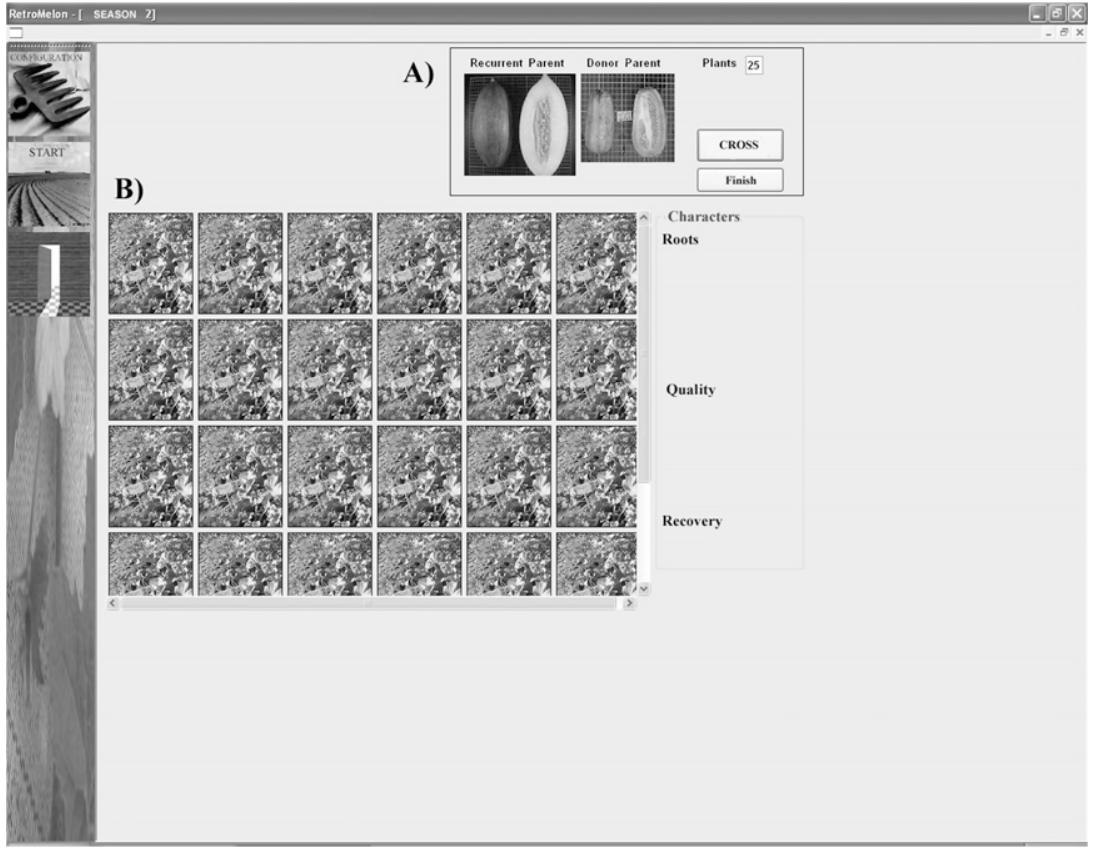

Fig. 2. Retromelon software screenshot showing different operations of the program. (A) Cross-selection area: this area displays the parentals and the number of individuals to be grown and phenotypically evaluated in the next generation. (B) Field visualization area, where the offspring from the cross-performed in (A) are displayed.
After making the first cross $(\operatorname{Pr} \times \mathrm{Pd})$, the software shows the $\mathrm{F}_{1}$ generation. In this generation, it is not necessary to make a selection for the next generation, as all the $F_{1}$ plants are expected to be genetically uniform and heterozygous for the trait under study (Kalloo, 1988). Students are allowed to discover the uniformity of the $\mathrm{F}_{1}$ generation by clicking on any of the plants (Fig. 2), all of which will display a similar appearance. The real selection begins at the first backcross generation. At this level, the students need to select which plants will be the parents for the next backcross generation according to the following: 1) incorporation of $\mathrm{Pd}$ resistance and 2 ) recovery of Pr agronomic traits. To simulate the process of phenotyping individual plants, the students must click on each plant to control the level of resistance and the recovery of $\operatorname{Pr}$ agronomic traits. The program will display a picture of a root system, a picture of a melon fruit, and a percentage of recovery of the Pr genome (Fig. 3). These images represent the phenotype of the plant on which the students base their selections. Thus, if a plant is very susceptible, the roots will appear damaged, whereas if a plant is resistant, it will show strong, healthy, and vigorous roots. The recovery of $\operatorname{Pr}$ agronomic

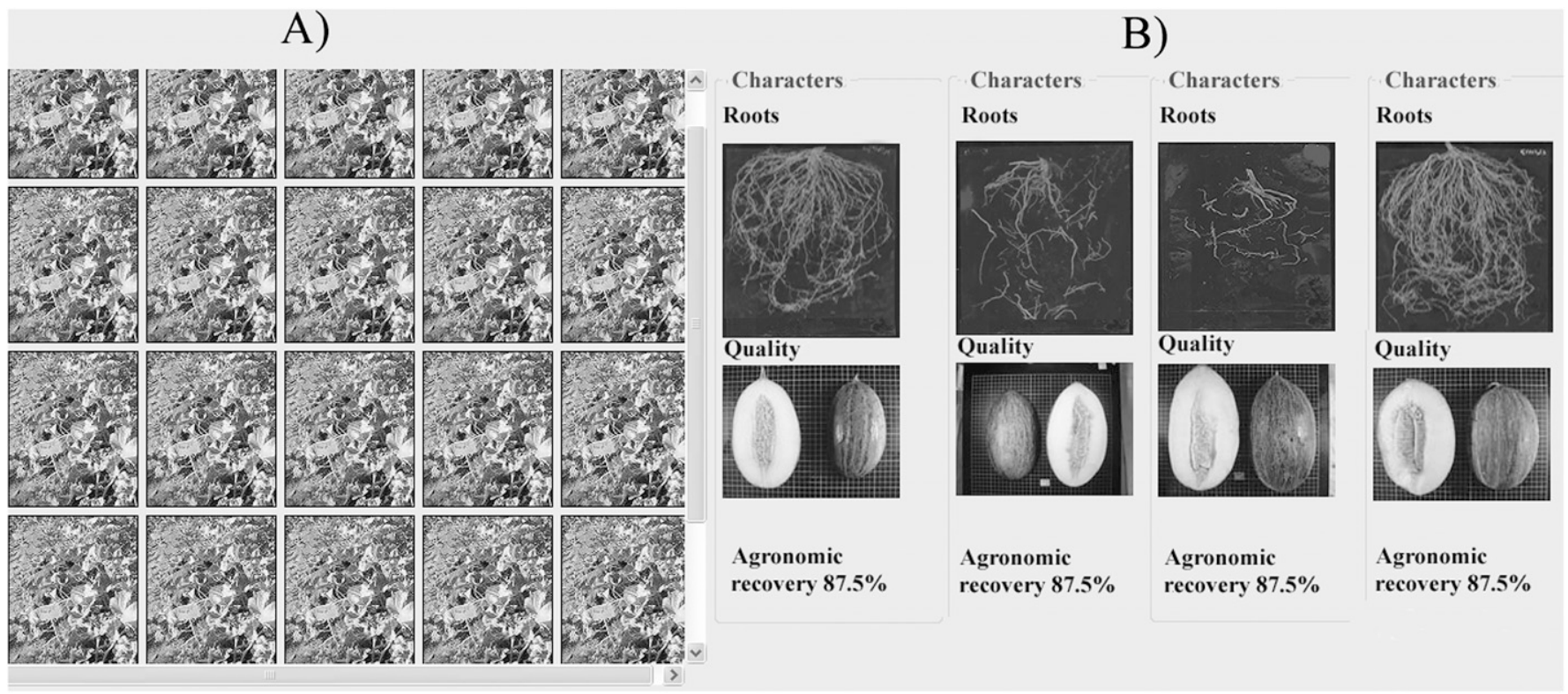

Fig. 3. Retromelon software screenshot. (A) Plants of a second backcross generation offspring; (B) root systems and fruits of four different plants selected (by clicking on them) from (A). Some plants show healthy and vigorous root systems [resistant to melon vine decline (MVD)], whereas others show rotted and broken roots (susceptible to MVD). On screen, the recovery of the recurrent parent genome is displayed as a percentage and as a fruit picture [lower row of pictures in (B)] with a certain resemblance to the recurrent parent. 


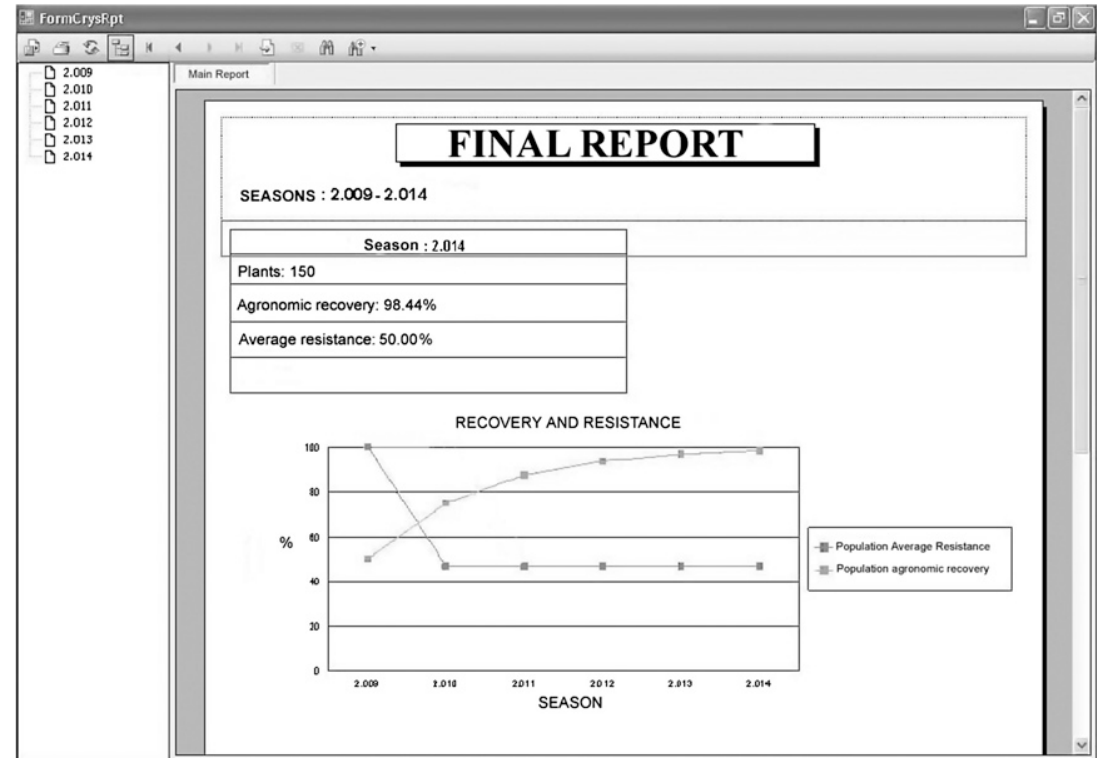

Fig. 4. Retromelon software screenshot of the final report indicating the following: 1) the number of cycles or seasons used in the breeding program, 2) the average resistance of the population, and 3 ) the average recovery of the genetic background of the recurrent parent.

features is visualized by displaying a picture of a melon fruit with a certain level of resemblance to the recurrent parent, according to the backcross generation (Fig. 3).

This evaluation-selection-cross cycle is repeated for several backcross generations until the students consider that the recovery of $\mathrm{Pr}$ agronomic appearance is high enough. At this point, the fixation of the resistance from $\mathrm{Pd}$ can be performed by selfing the selected individuals. Ultimately, the program generates a final report (Fig. 4) in which the student can observe the evolution of the integration of the resistance, the evolution of the Pr genome recovery, and how many cycles of cultivation were needed to achieve a certain degree of improvement.

\section{Results and discussion}

Currently, there are different software programs available for plant breeding programs. Most of them are mere data management tools, analysis tools, or both that are useful for breeders but with limited utility in teaching. One of the most complete web pages with software programs suitable for teaching purposes is that of the Wageningen University and Research Center (2010), which has several software programs aimed at assisting students to learn plant breeding. However, the only software program related to the backcross method, BCSim, is still in development and will be mostly suitable for more advanced students as it is focused on the evaluation and prediction of the results of marker-assisted backcross programs for the introgression of quantitative trait loci. Retromelon is directed at students of basic, general plant breeding courses.

Retromelon has several features that help students to integrate the knowledge transmitted in class lectures and emphasize points we consider to be crucial for understanding plant breeding. First, only by clicking on each plant of an offspring one is able to know the level of resistance of the plant. In a real breeding program, the plant breeder must evaluate every plant to select the best ones and then continue with the breeding program. Therefore, by clicking on each plant to visualize the phenotype, the student realizes the effort involved in phenotyping. Second, the student does not visualize the genetic configuration of the plants as the phenotype is the only information available to him or her. It is particularly of interest to show that the breeder deals with the phenotypes and needs to make decisions about them according to his or her previous knowledge of the genetic control of the gene. This idea also contributes to the understanding of the importance of molecular marker-based selection (Acquaah, 2007; Rodríguez-Burruezo et al., $2009 \mathrm{~b}$ ). Third, during the backcross breeding program, the student can try different options and make alternate decisions, even if they result in the loss of the resistance alleles. The software does not stop and does not correct the mistakes, so even if the student chooses the wrong parent to perform a cross, he or she will see the results in the next generation, as would happen in a real program. Crossing mistakes in real programs result in the loss of time and may ruin several years' work. If a student loses the resistance because of poor parent selection, he or she will have to end the program and start again. With the final report, the students can visualize their results and know how many cycles or years of real time are needed for a breeding program. The report also serves as a basis for discussion and comparison with the results and experiences of the students' classmates.

This program has been tested since 2007-2008 in applied genetics and plant breeding courses at the Universidad Politécnica de Valencia with undergraduate students, starting with an initial trial version with part of the students and, in subsequent years, expanding its use with all the students. The laboratory session in which students use the Retromelon software lasts $2 \mathrm{~h}$ and takes place after explaining the fundamentals of the backcross method in the classroom. At the beginning of the laboratory session, the students are asked to write a schematic of the two main types of backcross programs (with a dominant and a recessive gene) to clarify the possible doubts and queries. Subsequently, we explain how the software program is used and ask students to perform the steps of a breeding program using this tool. We encourage students to conduct several tests to try all possible options and observe their results on the final report. We later conduct a group discussion about the results and the entire process.

The three key points in learning how to perform a plant breeding program lie in understanding the following aspects: 1) the genetic control of the traits, 2) the importance of phenotyping, and 3) crop sexual biology and its consequences on the genetic configuration of the resulting population (Acquaah, 2007; Kalloo, 
Table 1. Performance of students in the examinations of the genetics and plant breeding course at the Universidad Politécnica de Valencia, Valencia, Spain.

\begin{tabular}{lccc}
\hline Academic yr & $\begin{array}{c}\text { Avg score on backcross } \\
\text { method exercise } \\
(\mathbf{0}-\mathbf{1} \text { scale })^{\mathrm{z}}\end{array}$ & $\begin{array}{c}\text { Avg score for } \\
\text { final exam } \\
(\mathbf{0}-\mathbf{1 0 ~ s c a l e})^{\mathrm{y}}\end{array}$ & $\begin{array}{c}\text { Students who } \\
\text { passed } \\
\text { the course (\%) }\end{array}$ \\
\hline $2005-2006^{\mathrm{x}}$ & $0.33 \mathrm{a}$ & $3.7 \mathrm{a}$ & 51 \\
$2006-2007^{\mathrm{x}}$ & $0.27 \mathrm{a}$ & $4.7 \mathrm{~b}$ & 72 \\
$2007-2008^{\mathrm{w}}$ & $0.27 \mathrm{a}$ & $5.1 \mathrm{~b}$ & 58 \\
$2008-2009^{\mathrm{v}}$ & $0.45 \mathrm{~b}$ & $6.8 \mathrm{~d}$ & 86 \\
$2009-2010^{\mathrm{v}}$ & $0.42 \mathrm{~b}$ & $5.8 \mathrm{c}$ & 82 \\
\hline
\end{tabular}

${ }^{2}$ The score of the exercise ranges from 0 to 1 , where 0 being a very bad answer, 0.5 enough to pass the exercise, and $\mathrm{l}$ an excellent answer.

'The score of the examination ranges from 0 to 10 , where 5-7 means passing the exam, 7-9 means an outstanding exam, and 9-10 means an extraordinary exam with high distinction.

"No use of Retromelon software in this course.

"First trial of the Retromelon software, used on a limited group of students.

"Use of Retromelon software with the whole class.

1988; Rodríguez-Burruezo et al., $2009 \mathrm{~b})$. These three points will determine the use of one plant breeding method or another and are highlighted when using Retromelon. The use of the software had a positive effect on students' understanding of the subject in exercises related to backcross breeding and on the results of their final exams. Students who used this software (years 2008-2009 and 20092010) showed fewer problems in understanding the backcross method and in schematizing it than students who did not use the software (Table 1). They also showed fewer problems in understanding other breeding methods and the real implications of plant breeding programs. This greater understanding is reflected in the increase of the final average score of the group in years 2008-2009 and 20092010 , when the software was used by all the students (Table $\mathrm{l}$ ).

We have identified several factors that may increase students' motivation when using Retromelon: 1) the work performed is very close to that of a real breeding program, 2) it integrates previous knowledge of the student, 3) it provides the opportunity to experiment many times without risk of failing, and 4) it can be used to refresh students' memory (Alonso-Tapia, 1998). These factors have been identified previously in the use of information technologies for horticulture teaching purposes (Boufford, 1994; Kahtz, 2000; VanDerZanden et al., 2008).

In the current context of university education, it is essential to have tools that allow students to develop the capacities necessary for their future career. In this sense, the major advantage of using simulators in the teaching of plant breeding is that they involve a practical application of skills that will subsequently be necessary throughout the students' career. Other skills developed or encouraged by the use of the Retromelon software include decision making, evaluation, and knowledge of the implications of work.

The program has English and Spanish versions and is available upon request to the authors for teaching purposes. So far, the software has been developed only for a backcross program. However, given the positive results and the clear benefits it brings to the learning of the subject, new simulators are being developed. These programs may introduce other plant breeding programs, such as mass selection, pedigree selection, plant breeding for asexually propagated plants, and selection for general or specific combining ability. Our experience has shown that this type of simulation software considerably improves undergraduate students' learning of plant breeding.

\section{Literature cited}

Acquaah, G. 2007. Principles of plant breeding and genetics. Blackwell, Malden, MA.

Alonso-Tapia, J. 1998. Motivar para el aprendizaje: Teoría y estrategias. Edebé, Barcelona, Spain.

Boufford, R.W. 1994. Using database management software to enhance learning in plant materials courses. Hort Technology 4:185-187.

Fita, A., B. Picó, and F. Nuez. 2007. Melon roots under stress: Melon vine decline. Plant Stress 1:93-104.
Fita, A., B. Picó, R.C.S. Dias, and F. Nuez. 2009a. 'Piel de Sapo' breeding lines tolerant to melon vine decline. HortScience 44:1458-1460.

Fita, A., C. Esteras, B. Picó, and F. Nuez. 2009b. Cucumis melo L. new breeding lines tolerant to melon vine decline. HortScience 44:2022-2024.

Kahtz, A.W. 2000. Can computer-assisted instruction be used by students for woody plant identification? HortTechnology 10:381-384.

Kalloo, G. 1988. Vegetable breeding. Vol. I. CRC Press, Boca Raton, FL.

Lea-Cox, J.D., C. Zhao, D.S. Ross, T.E. Bilderback, J.R. Harris, S.D. Day, C. Hong, T.H. Yeager, R.C. Beeson, W.L. Bauerle, A.G. Ristvey, M. Lorscheider, S. Dickinson, and J.M. Ruter. 2010. A nursery and greenhouse online knowledge center: Learning opportunities for sustainable practice. HortTechnology 20: 509-517.

Nakhlek, M.B. and J.S. Krajcik. 1993. A protocol analysis of the influence of technology on students' actions, verbal commentary, and thought processes during the performance of acid-base titrations. J. Res. Sci. Teach. 30:1149-1168.

Prohens, J., A. Rodríguez-Burruezo, C. Gisbert, S. Soler, F.J. Herraiz, M. Plazas, and A. Fita. 2010. Use of Capsicum and eggplant resources for practical classes of genetics and plant breeding courses. Proc. XIV EUCARPIA Meeting on Genetics and Breeding of Capsicum and Eggplant. 67-76.

Rodríguez-Burruezo, A., A. Fita, and J. Prohens. 2009a. New protocols to enhance learning of genetics and plant breeding in students of university degrees in agriculture. Proc. International Technology, Education and Development Conf. 4428-4433.

Rodríguez-Burruezo, A., J. Prohens, and A. Fita. 2009b. A primer of genetics and plant breeding. Universitat Politècnica València, València, Spain.

St. Hilaire, R., T.W. Sammis, and J.G. Mexal. 2009. Integrating hoop house construction and operation into an undergraduate general education horticulture class. HortTechnology 19:445-451.

VanDerZanden, A.M., D. Sandrock, and D. Kopsell. 2008. Student attitudes and perceptions of an online problem-based learning case study in landscape management. Hort Technology 18:520-523.

Wageningen University and Research Center. 2010. Genetics \& breeding related software which is available for download. 13 Sept. 2010. <http://www. plantbreeding.wur.nl/UK/software.html>. 